\title{
Editorial
}

\section{Infertility Associated to Endometriosis: Clarifying Some Important Controversies}

\author{
Paula Andrea Navarro ${ }^{10}$ \\ ${ }^{1}$ Department of Obstetrics and Gynecology, Faculdade de Medicina \\ de Ribeirão Preto da Universidade de São Paulo, Ribeirão Preto, SP, \\ Brazil \\ Rev Bras Ginecol Obstet 2019;41:523-524.
}

Endometriosis is an estrogen-dependent gynecological disease characterized by the presence and growth of endometrial tissue (glands and/or stroma) outside the uterine cavity. ${ }^{1}$ The disease affects $\sim 10 \%$ of women of reproductive age ${ }^{2}$ and is strongly associated with infertility. ${ }^{3}$ It is estimated that more than $30 \%$ of infertile women have endometriosis ${ }^{2}$ and that 30 to $50 \%$ of these women report difficulties in getting pregnant. ${ }^{4}$

Opinion remains divided as to whether minor endometriosis (minimal and mild endometriosis-stages I and II, respectively) has an adverse effect on the likelihood of conception..$^{5-9}$ In 1998 , a study demonstrated that the fecundity of infertile women with minimal or mild endometriosis is not significantly lower than that of women with unexplained infertility, suggesting that the initial stage of the disease is just a finding and not the cause of infertility. ${ }^{6}$ Otherwise, the findings of a randomized controlled trial showing improved natural conception rates following surgical treatment of visible endometriotic lesions suggest that the presence of visible minor lesions alone may have an adverse effect on natural conception. ${ }^{7}$ Additionally, a retrospective study of 192 fully investigated infertile couples (117 women with unexplained infertility and 75 with minimal/mild endometriosis without adhesive disease, both managed conservatively after diagnostic laparoscopy) evaluated cumulative pregnancy rates in both groups followed up for up to 3 years following laparoscopy. ${ }^{8}$ This study demonstrated that women with endometriosis had a lower probability of pregnancy compared with women with unexplained infertility (36\% versus $55 \%$, respectively), which confirmed the presence of lower cumulative pregnancy rates in women in the early stages of endometriosis compared with women with infertility of unknown cause, ${ }^{9}$ thus supporting the association between infertility and endometriosis in the early stages.

Infertility presented by women with early endometriosis, in whom pelvic anatomical distortions are not present, raises

(D) Paula Andrea Navarro's ORCID is https://orcid.org/0000-00032368-4188.

Address for correspondence Paula Andrea Navarro, Av. Bandeirantes 3900, 14049-900, Vila Monte Alegre, Ribeirão Preto, SP, Brazil (e-mail: drapaulanavarro @gmail.com). questions about the mechanisms involved in the impairment of fertility in patients with the disease. The research group under my supervision has extensively performed studies in this subject and published recent review articles approaching this topic. ${ }^{10-12}$ Although the mechanisms involved in endometriosis-related infertility are still not completely understood, in summary, there are studies suggesting the peritoneal, follicular, systemic, and endometrial microenvironments may be altered in these women, with consequent damages to folliculogenesis, oocyte quality, endometrial receptivity, and, even, sperm function. ${ }^{10-12}$

Another very controversial point is whether the indication of in-vitro fertilization (IVF) in infertile women with endometriosis is associated with a worse gestational prognosis after the procedure, and if the progression in disease staging further worsens the results of the IVF.

A systematic review published in $2002^{13}$ showed that patients with endometriosis who underwent ovarian stimulation for IVF had lower pregnancy rates when compared with infertile patients with tubal factor (relative risk (RR) 0.56, 95\% confidence interval [95\% CI] 0.44-0.70). However, when dividing endometriosis patients according to the staging of the disease (minimal/mild endometriosis-stages I/II and moderate/severe endometriosis-stages III/IV), it was observed that patients with endometriosis I/II presented pregnancy rates similar to those with tubal factor (RR $0.79,95 \% \mathrm{CI} 0.6-1.03$ ), and patients with endometriosis III/IV had significantly lower rates than those without the disease (RR $0.46,95 \% \mathrm{CI}$ $0.28-0.74$ ). Despite being a relevant review, its main limitation is that most of the included studies were published between 1980 and 1999, a period in which ovarian stimulation and technical conditions were quite different from the current ones.

However, more recent studies contradict the results of this meta-analysis published in 2002. Data from the latest published meta-analysis, ${ }^{14}$ which analyzed 36 cohort studies and randomized controlled trials, show that compared with women without endometriosis, women with endometriosis undergoing IVF and intracytoplasmic sperm injection (ICSI) had a

Copyright (c) 2019 by Thieme Revinter Publicações Ltda, Rio de Janeiro, Brazil
DOI https://doi.org/ 10.1055/s-0039-1697638. ISSN 0100-7203.
License terms

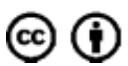


similar live birth rate per woman $(R R=0,94,95 \%$ CI 0.84-1.06, 13 studies, 12,682 patients, $I 2=35 \%$ ), lower clinical pregnancy rate per woman $(\mathrm{RR}=0.78,95 \% \mathrm{CI} 0.65-0.94)$ (mean difference of $-1.98,95 \%$ CI, 22.87-21.09, 17 studies, 17.593 cycles, $\mathrm{I} 2=97)$, a lower mean number of oocyte retrieved per cycle (mean difference - 1,98, 95\% CI - 2.87 to - 1.09, 17 studies, 17,593 cycles, $I 2=97 \%$ ). When compared with women without endometriosis, women with more advanced disease (stages III-IV) have lower rates of live births, clinical pregnancy rates, and mean number of retrieved oocytes. Briefly, data from this meta-analysis show a lower number of oocytes captured in women with endometriosis compared with women without endometriosis, and lower live birth rates only in women with stage III/IV endometriosis when compared with women without endometriosis.

It is important to state that women with advanced endometriosis have higher risk of presenting lower ovarian reserve than women without endometriosis, which may be related to the worse live birth rates demonstrated by this meta-analysis. Corroborating this hypothesis, a study published by our group evaluated 787 women who underwent IVF/ICSI (241 with endometriosis and 546 without) and demonstrated that although the mean age was similar between women with and without the diagnosis of endometriosis ( $33.8 \pm 4$ versus $33.7 \pm 4.4$ years, respectively), poor ovarian reserve, defined as an antral follicle count $\leq 6$ before the beginning of ovarian stimulation, was more common in women with endometriosis (39.8\% versus $22.7 \%$ ). ${ }^{15}$ The chance of achieving live birth was similar between women with the diagnosis of endometriosis and those without it (19.1\% versus $22.5 \%$ ), and also when considering only women with a poor ovarian reserve $(9.4 \%$ versus $8.9 \%$ ) and only those with a normal ovarian reserve ( 25.5 versus $26.5 \%$ ). Thus, we concluded that women diagnosed with endometriosis are more likely to have a poor ovarian reserve; however, their chance of conceiving by IVF/ICSI is similar to the one observed in patients without endometriosis and with a comparable ovarian reserve.

In conclusion, although still a controversial theme, it seems even minor endometriosis has an adverse effect on the likelihood of natural conception. The treatment of infertility associated with endometriosis has to be individualized, taking into consideration some important aspects, such as the age of the patient, her ovarian reserve, the stage of the disease, the presence of pelvic pain, endometrioma and previous surgical intervention, the presence or absence of tubal abnormality, and the seminal quality of the partner. When IVF is indicated, recent data show that the chance of conceiving is similar to the one observed in patients without endometriosis and with a comparable ovarian reserve.

\section{Conflicts of Interest}

The authors have no conflicts of interest to declare.

\section{References}

1 Giudice LC, Kao LC. Endometriosis. Lancet 2004;364(9447):1789-1799. Doi: 10.1016/S0140-6736(04)17403-5

2 Augoulea A, Alexandrou A, Creatsa M, Vrachnis N, Lambrinoudaki I. Pathogenesis of endometriosis: the role of genetics, inflammation and oxidative stress. Arch Gynecol Obstet 2012;286(01): 99-103. Doi: 10.1007/s00404-012-2357-8

3 Sensky TE, Liu DT. Endometriosis: associations with menorrhagia, infertility and oral contraceptives. Int J Gynaecol Obstet 1980;17 (06):573-576. Doi: 10.1002/j.1879-3479.1980.tb00210.x

4 Bulletti C, Coccia ME, Battistoni S, Borini A. Endometriosis and infertility. J Assist Reprod Genet 2010;27(08):441-447. Doi: 10.1007/s10815-010-9436-1

5 Revised American Society for Reproductive Medicine classification of endometriosis: 1996. Fertil Steril 1997;67(05):817-821. Doi: 10.1016/S0015-0282(97)81391-X

6 Bérubé S, Marcoux S, Langevin M, Maheux R; The Canadian Collaborative Group on Endometriosis. Fecundity of infertile women with minimal or mild endometriosis and women with unexplained infertility. Fertil Steril 1998;69(06):1034-1041. Doi: 10.1016/S0015-0282(98)00081-8

7 Marcoux S, Maheux R, Bérubé S; Canadian Collaborative Group on Endometriosis. Laparoscopic surgery in infertile women with minimal or mild endometriosis. N Engl J Med 1997;337(04): 217-222. Doi: 10.1056/NEJM199707243370401

8 Akande VA, Hunt LP, Cahill DJ, Jenkins JM. Differences in time to natural conception between women with unexplained infertility and infertile women with minor endometriosis. Hum Reprod 2004;19(01):96-103. Doi: 10.1093/humrep/deh045

9 D'Hooghe TM, Debrock S, Hill JA, Meuleman C. Endometriosis and subfertility: is the relationship resolved? Semin Reprod Med 2003;21(02):243-254. Doi: 10.1055/s-2003-41330

10 Da Broi MG, Navarro PA. Oxidative stress and oocyte quality: ethiopathogenic mechanisms of minimal/mild endometriosis-related infertility. Cell Tissue Res 2016;364(01):1-7. Doi: 10.1007/s00441015-2339-9

11 Da Broi MG, Giorgi VSI, Wang F, Keefe DL, Albertini D, Navarro PA. Influence of follicular fluid and cumulus cells on oocyte quality: clinical implications. J Assist Reprod Genet 2018;35(05):735-751. Doi: 10.1007/s10815-018-1143-3

12 Broi MGD, Ferriani RA, Navarro PA. Ethiopathogenic mechanisms of endometriosis-related infertility. JBRA Assist Reprod 2019;23 (03):273-280. Doi: 10.5935/1518-0557.20190029

13 Barnhart K, Dunsmoor-Su R, Coutifaris C. Effect of endometriosis on in vitro fertilization. Fertil Steril 2002;77(06):1148-1155

14 Hamdan M, Omar SZ, Dunselman G, Cheong Y. Influence of endometriosis on assisted reproductive technology outcomes: a systematic review and meta-analysis. Obstet Gynecol 2015;125 (01):79-88. Doi: 10.1097/AOG.0000000000000592

15 Coelho Neto MA, Martins WdeP, Luz CM, Jianini BT, Ferriani RA, Navarro PA. Endometriosis, ovarian reserve and live birth rate following in vitro fertilization/intracytoplasmic sperm injection. Rev Bras Ginecol Obstet 2016;38(05):218-224. Doi: 10.1055/s0036-1584126 